\title{
Study on Extraction Process of flavonoids in Mollugo Pentaphylla by Microwave-ultrasonic Extraction with Semi-bionic Technology
}

\author{
Liu Haijun ${ }^{a}$, Gao Yuan ${ }^{b}$ and Liu Keyue ${ }^{c *}$ \\ Jiujiang University, Jiujiang, Jiangxi,chaina \\ aemail:392507102@qq.com, bemail: cather_jju@126.com, ${ }^{c^{*}}$ email:elliee12345@aliyun.com
}

Keywords: Flavonoids; Microwave; Ultrasonic wave;Semi-bionic technology; Mollugo pentaphylla

\begin{abstract}
Objective: To optimize the extraction process of flavonoids in mollugo pentaphylla with microwave-ultrasonic extraction combination to semi-bionic technology by the response surface method. Methods: With the yield of flavonoids as response value, Extraction Process conditions of flavonoids in Mollugo Pentaphylla were investigated by the method of Box-Benhnken to analyze experiment. Results: The fitting degree of the regression model is high, and the optimum technological conditions were as follows, the material liquid ratio 1:30, microwave power $200 \mathrm{~W}$, extraction time $60 \mathrm{~s}$, and the ethanol concentration $60 \%$. Conclusion: The microwave-ultrasonic extraction with semi-bionic technology can be used for the extracting of flavonoids in mollugo pentaphylla.
\end{abstract}

\section{Introduction}

Use italic for emphasizing a word or phrase. Do not use boldface typing or capital letters except for section headings (cf. remarks on section headings, below). A Mollugo pentaphylla belongs to family molluginaceae, which has the efficacy of detoxification, antibacterial and dampness. It spreads in Jiangxi province and South of China. The whole plant can be used as medicine. Modern pharmacological studies found that the Mollugo pentaphylla extracts can significantly against arrhythmia induced by aconitine, chloroform and adrenaline. It has the pharmacological effect of against cardiovascular disease, such as crown expansion, anti-hypertension, etc ${ }^{[1-5]}$. The flavonoids content is abundant in Mollugo pentaphylla ${ }^{[6-7]}$. Numerous studies showed that the flavonoids have good efficacy in treating the diseases caused by oxidation, such as cardiovascular disease, cancer, inflammatory, caducity, etc ${ }^{[8-9]}$. Therefore, the flavonoids in mollugo pentaphylla may be the active substance to treat the cardiovascular and cerebrovascular diseases.

At present, the extraction methods of plant flavonoids include the organic solvent extraction, ethanol extraction, enzymatic, bionic extraction, microwave-assisted extraction, ultrasonic-assisted extraction, etc ${ }^{[10]}$. The researches showed that each method has its advantages and limitations, therefore, combination extraction technology is worth further studying. This paper focuses on the flavonoids in mollugo pentaphylla, adopts the microwave-ultrasonic extraction with Semi-bionic technology to extract flavonoids, and then employs the response surface method to optimize the extraction process, in order to explore a way of high efficient in extracting and reserving more components so as to better develop and utilize the flavonoids in mollugo pentaphylla and then generalized to the flavonoids extraction in other plants.

\section{Materials}

The mollugo pentaphylla collected from surrounding areas of Mount Lu, and formally assessed by professor Gao Chunhua. Rutin is produced by Sinopharm Chemical Reagent Co., Ltd. The ethanol, sodium nitrite, aluminum nitrate, sodium hydroxide, and hydrochloric acid used in the experiment are analytically pure. Ultrasound-Microwave Synergistic extraction device (CW2000, Shanghai New Extension Analytical Instruments Co., Ltd.), multi-purpose water circulation pump (SHZ-CH, Gongyi 
Yuhua Instrument Co., Ltd.), electronic analytical balance (BSA124S, Sartorius Scientific Instrument Co., Ltd.), high-speed grinder (QE-200 grams, Wuyi Yili Instrument Co., Ltd.), UV - visible spectrophotometer (UV-1100, Shanghai Mapada instrument Co., Ltd.).

\section{Methods}

Processing of Raw Materials. Dry and smash the mollugo pentaphylla, sift in 16 mesh, and reserve it.

Manufacture the Rutin Standard Curve. Dissolve $20 \mathrm{mg}$ standard rutin substance in $70 \%$ ethanol to $50 \mathrm{ml}$, shake up and obtain the $0.4 \mathrm{mg} / \mathrm{ml}$ rutin standard solution. Drip $0.00 \mathrm{ml}, 1.00 \mathrm{ml}, 2.00 \mathrm{ml}$, $3.00 \mathrm{ml}, 4.00 \mathrm{ml}$ and $5.00 \mathrm{ml}$ standard stock solution respectively to the $25 \mathrm{ml}$ volumetric flasks. First add $0.30 \mathrm{ml} 5 \%$ sodium nitrate, shake up and place for $6 \mathrm{~min}$; then drip $0.30 \mathrm{ml} 10 \%$ aluminum nitrate solution, shake up and place for $6 \mathrm{~min}$; finally, drip $4.00 \mathrm{ml} 0.1 \mathrm{~mol} / \mathrm{L}$ sodium hydroxide solution and $70 \%$ ethanol, shake up and place for $15 \mathrm{~min}$. UV-spectrophotometer is used to measure the absorbance value in $510 \mathrm{~nm}$ with blank solution as reference. Based on the data, the equation of linear regression is that $\mathrm{Y}=0.0091 \mathrm{X}-0.0053, \mathrm{R}^{2}=0.9994$; in which $\mathrm{X}$ stands for the rutin concentration and $\mathrm{Y}$ stands for the absorbance value.

The Determination of Flavonoids Content. Drip $1.00 \mathrm{ml}$ or $2.00 \mathrm{ml}$ extraction solution to the 25 $\mathrm{ml}$ volumetric flask, dissolve with ethanol to $5 \mathrm{ml}$. Add $0.3 \mathrm{ml} 5 \%$ sodium nitrite, shake up and place for $6 \mathrm{~min}$, then add $0.3 \mathrm{ml} \mathrm{10 \%}$ aluminium nitrate, shake up and place for another 6 min. Finally, add 4 $\mathrm{ml} 0.1 \mathrm{~mol} / \mathrm{L}$ sodium hydroxide, shake up and place for $15 \mathrm{~min}$. In this way, the total flavonoids content can be worked out.

The yield of total flavonoids content $=\mathrm{c} \times \mathrm{V} \times \mathrm{N} / \mathrm{w} \times 100 \%$

In this formula, "c" stands for the mass concentration of total flavonoids (mg/ml), "V" stands for the volume of crude extract $(\mathrm{ml})$, " $\mathrm{N}$ " stands for the dilution ratio, and "w" stands for the weight of raw material $(\mathrm{g})$.

Design on the Single Factor Experiment. The preliminary experiment demonstrates that the PH values of flavonoids which extracted by semi-bionic technology are 2.0-2.2, 7.0-8.0 in the first and second time respectively; and the factors influencing the yield of the flavonoids are liquid ratio, ethanol concentration, microwave power and extraction time. In this experiment, the liquid ratio is set as 1:20, $1: 25,1: 30,1: 35$, and 1:40; the ethanol concentration is set as $40 \%, 50 \%, 60 \%, 70 \%$, and $80 \%$; the microwave power is set as $100 \mathrm{~W}, 150 \mathrm{~W}, 200 \mathrm{~W}, 250 \mathrm{~W}$, and $300 \mathrm{~W}$; the extraction time is set as $120 \mathrm{~s}$, 180s, 240s, 300s, and 360s; and then single factor experiment is conducted respectively.

Design on the Response Surface Method to Optimize the Extraction Process of flavonoids. Using the Design Expert software, this experiment adopts the Box-Behnken to design the major extraction factors in the flavonoids' extraction process.

\section{Results and Analysis}

Impact of Liquid Ratio to the Yield of Flavonoids in Mollugo Pentaphylla. From figure 1, the yield of flavonoids in mollugo pentaphylla increases with the increase of liquid ratio, and the yield is the highest when the liquid ratio is 1:35. Considering the cost and convenience of the actual operation process, we should ensure the extraction effects while minimize the amount of solvent; hence, we determine the liquid ratio as 1:35, and then conduct subsequent optimization experiments.

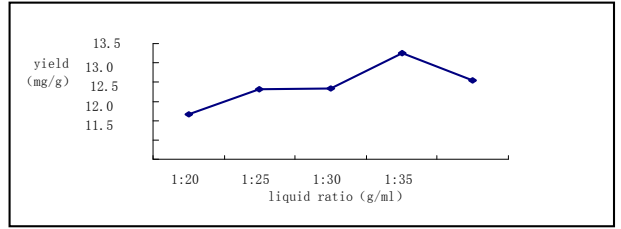

Figure. 1 Impact of Liquid Ratio to the Yield of Flavonoids in Mollugo Pentaphylla

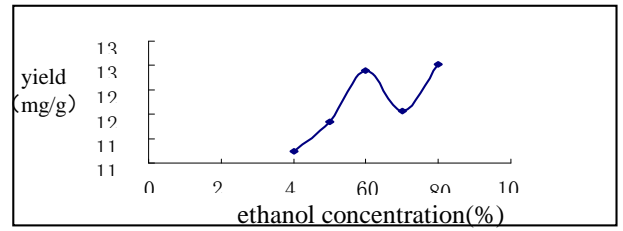

Figure. 2 Impact of Ethanol Concentration to the Yield of Flavonoids in Mollugo Pentaphylla 
Impact of Ethanol Concentration to the Yield of Flavonoids in Mollugo Pentaphylla . From figure 2, the yield of flavonoids in mollugo pentaphylla increases with the increase of ethanol concentration, and the yield is the highest when the concentration is $60 \%$; and then the yield decreases and increased again. We determine the ethanol concentration as $60 \%$ from economic point of view, and then conduct subsequent optimization experiments.

Impact of Microwave Power to the Yield of Flavonoids in Mollugo Pentaphylla. From figure 3, with the increase of microwave power, the yield of flavonoids in mollugo pentaphylla increases first and then decreases. The flavonoids may decompose with the increase of microwave power in later stage. Therefore, we determine the microwave power as $250 \mathrm{~W}$, and then conduct subsequent optimization experiments.

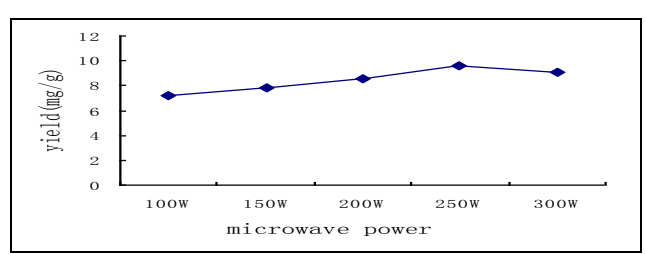

Figure. 3 Impact of Microwave Power to the Yield of Flavonoids in Mollugo Pentaphylla

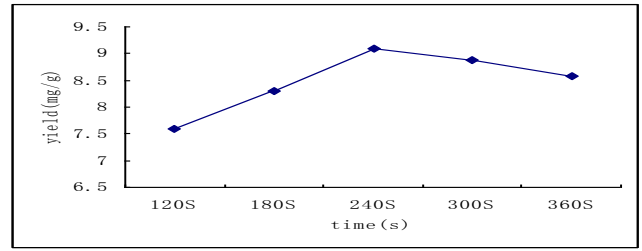

Figure. 4 Impact of Microwave Time to the Yield of Flavonoids in Mollugo Pentaphylla

Impact of Microwave Time to the Yield of Flavonoids in Mollugo Pentaphylla. From figure 4, with the increase of microwave time, the yield of flavonoids in mollugo pentaphylla increases first and then decreases. The non-flavonoids compositions may dissolve with the increase of microwave time. Therefore, we determine the microwave time as $240 \mathrm{~S}$, and then conduct subsequent optimization experiments.

\section{Adopt Response Surface Method to Optimize the Extraction Process Condition}

Design of Response Surface Experiment and Results. On the basis of single factor experiment, select liquid ratio, microwave power, extraction time, and ethanol concentration as independent variables, and design the response surface with four factors and three levels according to the principle of Box-Benhnken. There are 29 experiment points, and the experimental results are shown as table 2.

Mathematical Modeling and Significance Testing. The data are multiple regression fitting, and the result of variance analysis is shown in table 3. The regression model is that $\mathrm{Y}=14.86+0.33 \mathrm{~A}-0.29 \mathrm{~B}$ $+0.13 \mathrm{C}+0.088 \mathrm{D}+0.29 \mathrm{AB}+0.022 \mathrm{~A} \mathrm{C}+0.21 \mathrm{~A} \mathrm{D}-0.23 \mathrm{BC}-0.57 \mathrm{BD}+0.75 \mathrm{CD}$. The model is significant, which indicates that the test designing is correct and can be used to predict the response values.

The significance test results of regression coefficient in secondary model indicate that $\mathrm{A}, \mathrm{B}$, and $\mathrm{BD}$ are significant to the extraction ratio, which manifests that it is not the simple linear relationship between the experimental factors and response values; and among the factors the influence of a factor is relatively small while the quadratic factors are more significant which are similar to the ones in regression equation.

Determine the Best Extraction Conditions. The optimization conditions are obtained by using the Design Expert software, and determine the optimal extraction process by microwave-assisted and semi-bionic technology: microwave power $200 \mathrm{~W}$, extraction time $300 \mathrm{~S}$, ethanol concentration $60 \%$, and liquid ratio 1:30. The theoretical calculation of flavonoids' extraction ratio is $13.867 \%$, The actual measured flavonoids' extraction ratio is $13.975 \%$ which is close to the predicted value.

\section{Conclusion}

Design experiments by using response surface method. According to the design principle of Box-Benhnken, four factors (microwave power, extraction time, ethanol concentration and liquid ratio) 
which are significant to the flavonoids' extraction ratio are chosen to analyze the experiments. The results indicate that the influence of above four factors to the extraction ratio is not the simple linear relationship.

The conditions in extraction process of flavonoids by microwave-ultrasonic extraction with semi-bionic technology are designed in response surface by using the Design Expert software. The results show that this method has good applicability and predictability, and can provide more accurate information on the variables and error. The optimum conditions are as follows: microwave power $200 \mathrm{~W}$, extraction time $300 \mathrm{~S}$, ethanol concentration $60 \%$, and liquid ratio 1:30.

\section{Fund Program}

National Natural Science Foundation of China (No: 81260606); Jiangxi Provincial Natural Science Foundation (No: 20122BAB205074); Jiangxi Province Science and Technology Project (No: 20141BBG70084); Project in Chinese Medicine of Jiangxi Health Department (No: 2010A148).

Table 1 Design of Response Surface Experiment and Results

\begin{tabular}{|c|c|c|c|c|c|c|}
\hline $\begin{array}{l}\text { Num } \\
\text { ber }\end{array}$ & $\mathrm{r}$ & $\begin{array}{l}\text { Powe } \\
(\mathrm{W})\end{array}$ & $\begin{array}{l}\text { Time } \\
(\mathrm{S})\end{array}$ & $\begin{array}{l}\text { Liquid ratio } \\
(1: X)\end{array}$ & $\begin{array}{ll} & \text { Concentratio } \\
\mathrm{n} & (\%)\end{array}$ & $\begin{array}{l}\text { Extraction ratio } \\
(\mathrm{mg} / \mathrm{g})\end{array}$ \\
\hline 1 & & 250 & 180 & 30 & 70 & 13.04508 \\
\hline 2 & & 200 & 180 & 30 & 60 & 13.50602 \\
\hline 3 & & 250 & 240 & 35 & 70 & 14.39736 \\
\hline 4 & & 300 & 240 & 35 & 60 & 13.88018 \\
\hline 5 & & 250 & 240 & 30 & 60 & 14.45266 \\
\hline 6 & & 250 & 300 & 30 & 50 & 14.71775 \\
\hline 7 & & 250 & 240 & 30 & 60 & 14.0509 \\
\hline 8 & & 250 & 240 & 30 & 60 & 13.97814 \\
\hline 9 & & 250 & 240 & 25 & 70 & 13.95601 \\
\hline 10 & & 200 & 240 & 30 & 50 & 14.86449 \\
\hline 11 & & 250 & 300 & 35 & 60 & 13.95435 \\
\hline 12 & & 250 & 180 & 30 & 50 & 13.90141 \\
\hline 13 & & 200 & 240 & 35 & 60 & 14.47935 \\
\hline 14 & & 200 & 240 & 25 & 60 & 14.41087 \\
\hline 15 & & 300 & 240 & 25 & 60 & 15.44551 \\
\hline 16 & & 200 & 240 & 30 & 70 & 14.22812 \\
\hline 17 & & 250 & 240 & 25 & 50 & 15.72689 \\
\hline 18 & & 250 & 180 & 25 & 60 & 15.18334 \\
\hline 19 & & 250 & 240 & 35 & 50 & 14.87317 \\
\hline 20 & & 300 & 180 & 30 & 60 & 14.53441 \\
\hline 21 & & 200 & 300 & 30 & 60 & 14.2448 \\
\hline 22 & & 300 & 300 & 30 & 60 & 14.96568 \\
\hline 23 & & 250 & 240 & 30 & 60 & 15.31696 \\
\hline 24 & & 250 & 180 & 35 & 60 & 15.70624 \\
\hline 25 & & 300 & 240 & 30 & 50 & 15.23537 \\
\hline 26 & & 250 & 300 & 25 & 60 & 14.36993 \\
\hline 27 & & 300 & 240 & 30 & 70 & 15.42211 \\
\hline 28 & & 250 & 240 & 30 & 60 & 14.93139 \\
\hline 29 & & 250 & 300 & 30 & 70 & 14.59955 \\
\hline
\end{tabular}


Table 2 Variance Analysis in Regression Model and Test of Significance

\begin{tabular}{lllll}
\hline Source & Squares & Square & Value & Prob $\gg \mathrm{F}$ \\
\hline Model & 6.95 & 0.70 & 3.54 & 0.0095 \\
A-A & 1.33 & 1.33 & 6.77 & 0.0180 \\
B-B & 1.04 & 1.04 & 5.28 & 0.0337 \\
C-C & 0.22 & 0.22 & 1.11 & 0.3067 \\
D-D & 0.092 & 0.092 & 0.47 & 0.5019 \\
AB & 0.34 & 0.34 & 1.74 & 0.2036 \\
AC & $1.896 \mathrm{E}-003$ & $1.896 \mathrm{E}-003$ & $9.659 \mathrm{E}-003$ & 0.9228 \\
AD & 0.17 & 0.17 & 0.86 & 0.3652 \\
BC & 0.22 & 0.22 & 1.12 & 0.3035 \\
BD & 1.31 & 1.31 & 6.67 & 0.0187 \\
CD & 2.24 & 2.24 & 11.39 & 0.0034 \\
Residual & 3.53 & 0.20 & & \\
Lack of Fit & 3.31 & 0.24 & 4.26 & 0.0857 \\
Pure Error & 0.22 & 0.055 & & \\
Cor Total & 10.49 & & & \\
\hline
\end{tabular}

\section{References}

[1] Yao Guoyuan, Li Li, et al. Experimental study of anti-arrhythmic effects of mollugo pentaphylla extract (MP876), J. Jiujiang Medical College. 1 (1992) 7-9.

[2] Yao Guoyuan, Lu Qihua, et al. The anti-aconitine of mollugo pentaphylla extract (MP876) and its impact on rat plasma and myocardial cAMP, J. Pharmacology and Clinics. 7 (1991) 11-16.

[3] Zhou Kaixuan, Chen Bo, et al. Preliminary experimental study on mollugo pentaphylla's cardiac pharmacological effects, J. Jiujiang Medical College. 1 (1989) 7-9.

[4] Yao Guoyuan, Lu Qihua, et al.. The anti-arrhythmic effects of mollugo pentaphylla to mice and its impact on plasma and myocardial cAMP, J. Jiujiang Medical College. 2 (1989) 12-14.

[5] Li Xueqin, Gao Chunhua, et al. Effect of mollugo pentaphylla on blood pressure in spontaneously hypertensive rats, J. Shandong Medical Journal. 6 (2005) 8-11.

[6] Liu Keyue, Liu Haijun, Qu Weihon, et al.. Virtual screening study of inhibiting TNF-aactive constituent in mullugo pentaphylla, J. Anhui Agricultural Sciences. 18 (2009) 8474, 8509

[7] Liu Keyue, Liu Haijun, Gao Chunhua, et al. Study on the chemical compositions in mollugo pentaphylla, J. Lishizhen Med Mater Med Res. 8 (2009) 1916-1917.

[8] Xu Xiaon, Niu Ziran, Wang Shoubao, et al. Effect and mechanism of total flavonoids of bugloss on rats with myocardial ischemia and reperfusion injury, J. Acta Pharmaceutica Sinica. 6 (2014):875-881.

[9] Zhou Jun, Zhou Desheng. Pharmacological study on the effects of flavonoids to the heart and cerebral vessels, J. Chinese Journal of Integrative Medicine on Cardio-/Cerebrovascular Disease. 6 (2010) 725-727. 
[10] Nie Yanhua, Bi Huimin, \& Lin Danqiong. Research and analysis of biological flavonoids extraction method, J. Food Engineering. 1(2013)3-5 